\title{
Nonclassicality of states and measurements by breaking classical bounds on statistics
}

\author{
Ángel Rivas* \\ School of Physics, Astronomy, and Mathematics, University of Hertfordshire, College Lane, Hatfield, Hertfordshire AL10 $9 A B$, \\ United Kingdom
}

\author{
Alfredo Luis ${ }^{\dagger}$ \\ Departamento de Óptica, Facultad de Ciencias Físicas, Universidad Complutense, 28040 Madrid, Spain
}

(Received 19 September 2008; revised manuscript received 1 February 2009; published 24 April 2009)

\begin{abstract}
We derive exceedingly simple practical procedures revealing the quantum nature of states and measurements by the violation of classical upper bounds on the statistics of arbitrary measurements. Data analysis is minimum, and definite conclusions are obtained without evaluation of moments or any other more sophisticated procedures. These nonclassical tests are independent of other typical quantum signatures such as subPoissonian statistics, quadrature squeezing, or oscillatory statistics. This approach can be equally well applied to very diverse situations such as single- and two-mode fields, observables with continuous and discrete spectra, finite- and infinite-dimensional systems, and ideal and noisy measurements.
\end{abstract}

DOI: 10.1103/PhysRevA.79.042105

PACS number(s): 03.65.Ta, 42.50.Dv, 03.65.Ca, 42.50.Ar

\section{INTRODUCTION}

Nonclassicality is a key concept supporting the necessity of the quantum theory [1-8]. A customary signature of nonclassical behavior is the failure of the Glauber-Sudarshan $P$ phase-space representation to exhibit all the properties of a classical probability density. This occurs when $P$ takes negative values or when it fails to be a proper function becoming a generalized function or distribution.

Within standard quantum theory, quantum states play two dissimilar but complementary roles: (i) they express the state of the system and (ii) they determine the statistics of measurements by projection on the system state, such as, for example, photon-number and quadrature measurements in quantum optics. We may refer to them as measured and measuring states, respectively.

In this work we derive exceedingly simple and robust practical procedures to reveal the quantum nature of measured and measuring states. In this regard, while characterization of nonclassical (measured) states has been well developed [1-6], much less attention has received the characterization of measurements [8]. One of the purposes of this work is to contribute to fill this gap addressing the characterization of nonclassical measurements, i.e., when the measuring state is nonclassical. More specifically, measurements are described by positive operator-valued measures (POVMs) $\Delta_{m}$, such that the statistics of the measurement is $p_{m}=\operatorname{tr}\left(\Delta_{m} \rho\right)$, where $\rho$ is the measured state. We will say that the measurement is nonclassical when the $P$ representative of some $\Delta_{m}$ takes negative values or is a generalized function. In most practical situations, $\Delta_{m}$ define legitimate measuring states $\rho_{m} \propto \Delta_{m}$ so that the measurement is nonclassical if and only if some $\rho_{m}$ is nonclassical. Nonclassicality of measurements has been recently related with the noncontextuality problem in Ref. [7].

\footnotetext{
*a.rivas@herts.ac.uk

†URL: http://www.ucm.es/info/gioq; alluis@ fis.ucm.es
}

The main contributions of this work are as follows:

(i) We derive exceedingly simple practical procedures that can reveal the quantum nature of states and measurements. These are upper bounds on measurement statistics which are satisfied by all states and measurements for which the $P$ representative is a non-negative function compatible with classical physics. The lack of compliance of these statistical bounds is thus a nonclassical signature.

(ii) This approach can be applied to arbitrary measurements, which may involve, for example, single- or two-mode electromagnetic fields, observables with continuous or discrete spectrum, systems on finite- or infinite-dimensional spaces, ideal or noisy measurements, etc. (Some of these possibilities are considered in detail below.) This is in sharp contrast with other nonclassical criteria that refer exclusively to specific measuring schemes.

(iii) A key point of this approach is that data analysis is reduced to minimum. At difference with other tests of nonclassical behavior, in our case definite conclusions can be obtained without evaluation of moments or any other more sophisticated data elaborations [1-6]. This is reflected on the robustness under practical imperfections that may even favor observation of nonclassical behavior.

(iv) These nonclassical tests are independent of other typical quantum signatures of nonclassical behavior such as subPoissonian statistics, squeezing, or oscillatory statistics [1]. To this end we propose examples of quantum states violating classical bounds that present no such typical quantum signatures.

To derive the nonclassical tests we will use the $P$ and $Q$ phase-space representatives associated to any operator $A$ defined as

$$
A=\int d^{2} \alpha P(\alpha)|\alpha\rangle\langle\alpha|, \quad Q(\alpha)=\frac{1}{\pi}\langle\alpha|A| \alpha\rangle,
$$

where $|\alpha\rangle$ are coherent states, $a|\alpha\rangle=\alpha|\alpha\rangle$, and $a$ is the annihilation or complex-amplitude operator. They are suitably normalized; 


$$
\int d^{2} \alpha P(\alpha)=\int d^{2} \alpha Q(\alpha)=\operatorname{tr} A,
$$

with $d^{2} \alpha=d x d y$, where $x$ and $y$ are the real and imaginary parts of $\alpha=x+i y$. The measured statistics $p_{m}=\operatorname{tr}\left(\Delta_{m} \rho\right)$ can be then expressed as

$$
p_{m}=\pi \int d^{2} \alpha P_{m}(\alpha) Q(\alpha)=\pi \int d^{2} \alpha P(\alpha) Q_{m}(\alpha),
$$

where $P(\alpha)$ and $Q(\alpha)$ are the $P$ and $Q$ representatives of the measured state $\rho$, while $P_{m}(\alpha)$ and $Q_{m}(\alpha)$ are the ones associated to the POVM $\Delta_{m}$.

In Secs. II and III we derive simple bounds to $p_{m}$ able to reveal the nonclassical nature of measuring states $\rho_{m} \propto \Delta_{m}$ and measured states $\rho$, respectively. The robustness of these criteria under practical imperfections is examined in Sec. IV. This formalism is further extended to two-mode situations in Sec. V and adapted to finite-dimensional systems in Sec. VI.

\section{NONCLASSICAL MEASUREMENTS}

From Eq. (1.3) we can derive classical bounds disclosing nonclassical measurements. For every ordinary non-negative function $P_{m}(\alpha) \geq 0$ it holds that for every $\alpha$

$$
P_{m}(\alpha) Q(\alpha) \leq P_{m}(\alpha) Q_{\max },
$$

where $Q_{\max }$ is the maximum of $Q(\alpha)$ [note that $Q(\alpha)$ is always a positive and well behaved function]. Applying this to the first equality in Eq. (1.3) we get the following upper bound for $p_{m}$ provided that $\operatorname{tr} \Delta_{m}$ is finite,

$$
p_{m} \leq \pi Q_{\max } \operatorname{tr} \Delta_{m} .
$$

Equation (2.2) can be violated if $P_{m}(\alpha)$ fails to be positive or when it becomes a generalized function. In both cases Eq. (2.1) fails to be true. Therefore, the violation of condition (2.2) is a signature of nonclassical measurement.

The existence of $P_{m}(\alpha)$ as a classical probability density for all $m$ allows us to understand the measurement as a classical stochastic process [9] between the phase space and the sample space, with transition probability kernel given by $K(m, \alpha)=\pi P_{m}(\alpha)$. Conversely, the failure of $K(m, \alpha)$ to be a classical conditional probability density denotes the quantum nature of the measurement process.

In order to detect the violation of the classical bound [Eq. (2.2)] the only prior information required about the measurement being performed is the $\operatorname{trace} \operatorname{tr}\left(\Delta_{m}\right)$. This can be measured using explicit practical methods (see some proposals in the Appendix). In any case this is not a very stringent condition since in most practical situations this can be inferred from simple rough analyses of the experimental arrangement by symmetry considerations, etc.

Note that coherent states $|\alpha\rangle$ are useless as measured states to reveal nonclassical measurements since $\pi Q_{\max }=1$ so that Eq. (2.2) leads to the trivial bound $p_{m} \leq \operatorname{tr} \Delta_{m}$ for all measurements [10]. This bound is trivial because, using the Cauchy-Schwarz inequality,

$$
\left|\operatorname{tr}\left(A B^{\dagger}\right)\right|^{2} \leq \operatorname{tr}\left(A A^{\dagger}\right) \operatorname{tr}\left(B B^{\dagger}\right),
$$

we get

$$
p_{m}^{2}=\left[\operatorname{tr}\left(\rho \Delta_{m}\right)\right]^{2} \leq \operatorname{tr}\left(\rho^{2}\right) \operatorname{tr}\left(\Delta_{m}^{2}\right),
$$

and using that for positive operators $\operatorname{tr}\left(A^{2}\right) \leq(\operatorname{tr} A)^{2}$ we get

$$
p_{m}=\operatorname{tr}\left(\rho \Delta_{m}\right) \leq \operatorname{tr} \rho \operatorname{tr} \Delta_{m}=\operatorname{tr} \Delta_{m} .
$$

Otherwise, quantum or classical state other than coherent may be used since the weight of the criteria relies on the behavior of $P_{m}(\alpha)$.

This approach is next illustrated with the examples of photon-number and field quadrature measurements performed on a single-mode electromagnetic field.

\section{A. Photon-number measurements}

In order to illustrate this formalism the simplest example is the ideal photon-number measurement, $\Delta_{n}=\rho_{n}=|n\rangle\langle n|$, where $|n\rangle$ are number states, $a^{\dagger} a|n\rangle=n|n\rangle$ so that $\operatorname{tr} \Delta_{n}=1$. In this case the classical bound in Eq. (2.2) becomes

$$
p_{n} \leq \pi Q_{\max }=p_{b},
$$

which is actually independent of the outcome $n$.

A readily demonstration of the nonclassical nature of the photon-number measurement is provided when $n=1$ and the measured state is the one-photon state $|n=1\rangle$. In such a case $p_{1}=1$

$$
Q(\alpha)=\frac{|\alpha|^{2}}{\pi} \exp \left(-|\alpha|^{2}\right), \quad \pi Q_{\max }=\frac{1}{e},
$$

where the maximum occurs for $|\alpha|=1$. Thus we have that

$$
p_{1}=1>\pi Q_{\max } \operatorname{tr} \Delta_{1}=\frac{1}{e}
$$

so that the measurement is nonclassical and the classical upper bound is surpassed by $172 \%$ since $\left(p_{1}-p_{b}\right) / p_{b}=1.72$.

\section{B. Quadrature measurements}

Concerning quadrature measurements (implemented in practice by homodyne detection [1]) we have $\Delta_{x}=|x\rangle\langle x|$, where $|x\rangle$ are the eigenstates of the quadrature operator

$$
X=\frac{1}{2}\left(a^{\dagger}+a\right), \quad X|x\rangle=x|x\rangle,
$$

being the optical analog of mechanical position or linear momentum. In this case $\operatorname{tr} \Delta_{x}$ is not finite since $|x\rangle$ are not normalizable $\left\langle x \mid x^{\prime}\right\rangle=\delta\left(x-x^{\prime}\right)$.

In order to avoid this difficulty we can appreciate that the $P$ representative of $|x\rangle\langle x|, P_{x}\left(\alpha=x^{\prime}+i y^{\prime}\right)$, does not depend on $y^{\prime}$. This is an observable property, for example, via the independence of statistics under displacements of the measured state along this coordinate. Thus we can rearrange Eq. (1.3) in the form

$$
p_{x}=\pi \int d x^{\prime} P_{x}\left(x^{\prime}\right) \tilde{Q}\left(x^{\prime}\right), \quad \tilde{Q}\left(x^{\prime}\right)=\int d y^{\prime} Q\left(x^{\prime}, y^{\prime}\right)
$$

so that Eq. (2.2) is replaced by 


$$
p_{x} \leq \pi \widetilde{Q}_{\max } \operatorname{tr}_{x} \Delta_{x},
$$

where $\widetilde{Q}_{\max }$ is the maximum of $\widetilde{Q}(x)$ when $x$ is varied, and

$$
\operatorname{tr}_{x} \Delta_{x}=\int d x^{\prime} P_{x}\left(x^{\prime}\right)=\int d x^{\prime} Q_{x}\left(x^{\prime}\right),
$$

where $P_{x}$ and $Q_{x}$ are the representatives of $\Delta_{x}$, with

$$
Q_{x}\left(x^{\prime}\right)=\frac{1}{\pi}\left|\left\langle x \mid \alpha=x^{\prime}+i y^{\prime}\right\rangle\right|^{2}=\frac{1}{\pi} \sqrt{\frac{2}{\pi}} \exp \left[-2\left(x-x^{\prime}\right)^{2}\right] .
$$

This leads to $\operatorname{tr}_{x} \Delta_{x}=1 / \pi$ and to the classical upper bound,

$$
p_{x} \leq \widetilde{Q}_{\max }=p_{b},
$$

that does not depend on the outcome $x$.

\section{Thermal-chaotic state}

In order to look for violations of bound (2.14) let us consider that the measured state $\rho$ is the thermal-chaotic state whose expression in photon-number basis is

$$
\rho_{\mathrm{tc}}=(1-\xi) \sum_{n=0}^{\infty} \xi^{n}|n\rangle\langle n|,
$$

where $\xi$ is a real parameter with $0 \leq \xi<1$. These states describe most classical light sources. The mean number of photons $n_{\mathrm{tc}}$ and the quadrature variance are

$$
n_{\mathrm{tc}}=\frac{\xi}{1-\xi}, \quad(\Delta X)^{2}=\frac{1}{4}\left(1+2 n_{\mathrm{tc}}\right),
$$

while the $Q$ and $\widetilde{Q}$ functions are

$$
\begin{gathered}
Q(\alpha)=\frac{1}{\pi\left(n_{\mathrm{tc}}+1\right)} \exp \left(-\frac{|\alpha|^{2}}{n_{\mathrm{tc}}+1}\right), \\
\widetilde{Q}\left(x^{\prime}\right)=\frac{1}{\sqrt{\pi\left(n_{\mathrm{tc}}+1\right)}} \exp \left(-\frac{x^{\prime 2}}{n_{\mathrm{tc}}+1}\right)
\end{gathered}
$$

so that the upper bound in Eq. (2.14) reads as

$$
p_{b}=\frac{1}{\sqrt{\pi\left(n_{\mathrm{tc}}+1\right)}} .
$$

The statistics of the quadrature measurement $p_{x}=\left|\left\langle x\left|\rho_{t c}\right| x\right\rangle\right|^{2}$ is Gaussian

$$
p_{x}=\frac{1}{\sqrt{2 \pi} \Delta X} \exp \left[-\frac{x^{2}}{2(\Delta X)^{2}}\right]
$$

and the output most likely to break bound (2.2) is $x=0$ since it maximizes $p_{x}$. This outcome will infringe the bound provided that

$$
p_{0}=\frac{1}{\sqrt{\pi\left(n_{\mathrm{tc}}+\frac{1}{2}\right)}}>p_{b}=\frac{1}{\sqrt{\pi\left(n_{\mathrm{tc}}+1\right)}},
$$

which holds for every $n_{\mathrm{tc}}$. In particular for $n_{\mathrm{tc}}=0$ (the vacuum state) we have $p_{0}=0.80$ and $p_{b}=0.56$ so that the classical upper bound is very clearly surpassed by $100\left(p_{0}\right.$ $\left.-p_{b}\right) / p_{b}=43 \%$.

The outputs $x$ that contravene Eq. (2.14) are all $x$ such that

$$
x^{2}<(\Delta X)^{2} \ln \left[1+\frac{1}{4(\Delta X)^{2}}\right] .
$$

For $n_{\mathrm{tc}}=0$ these are all $x$ in the interval $-0.42 \leq x \leq 0.42$, which occur with a $60 \%$ probability since $\int_{-0.42}^{0.42} p_{x} d x \simeq 0.60$.

\section{Squeezed vacuum}

As a further example, when the measured state is the squeezed vacuum the quadrature statistics has again the Gaussian form [Eq. (2.19)], being the $Q$ function

$$
Q(x, y)=\frac{1}{\pi} \frac{4 \Delta X}{1+4(\Delta X)^{2}} \exp \left[-\frac{2 x^{2}+8(\Delta X)^{2} y^{2}}{1+4(\Delta X)^{2}}\right]
$$

so that

$$
\tilde{Q}(x)=\sqrt{\frac{2}{\pi\left[1+4(\Delta X)^{2}\right]}} \exp \left[-\frac{2 x^{2}}{1+4(\Delta X)^{2}}\right]
$$

and

$$
\tilde{Q}_{\max }=\sqrt{\frac{2}{\pi\left[1+4(\Delta X)^{2}\right]}} .
$$

The output most likely to break bound (2.14) is $x=0$, and in such a case the classical bound is surpassed for all $\Delta X$ since

$$
p_{0}=\frac{1}{\sqrt{2 \pi} \Delta X}>p_{b}=\sqrt{\frac{2}{\pi\left[1+4(\Delta X)^{2}\right]}} .
$$

The most favorable situation is when $\Delta X$ is as small as possible. For example, for $\Delta X=0.1$ we have $p_{0}=4.0$ and $\widetilde{Q}_{\max }$ $=0.8$ so that there is a percentage of violation of $100\left(p_{0}\right.$ $\left.-p_{b}\right) / p_{b}=400 \%$ approximately. The output $x$ 's that contravene Eq. (2.14) are given by Eq. (2.21), which for $\Delta X=0.1$ is the interval $-0.18 \leq x \leq 0.18$ that represents the $93 \%$ of all outcomes since $\int_{-0.18}^{0.18} p_{x} d x \simeq 0.93$.

\section{NONCLASSICAL STATES}

In this section we derive classical bounds disclosing nonclassical measured states. They can be derived from the last equality in Eq. (1.3) by considering that for classical states, i.e., for ordinary non-negative functions $P(\alpha) \geq 0$, we get

$$
P(\alpha) Q_{m}(\alpha) \leq P(\alpha) Q_{m, \max },
$$

where $Q_{m, \max }$ is the maximum of $Q_{m}(\alpha)$. Applying this to the last equality in Eq. (1.3) and taking into account Eq. (1.2), we get the following upper bound for $p_{m}$,

$$
p_{m} \leq \pi Q_{m, \max },
$$

that holds for every $P(\alpha)$ compatible with classical physics. If this condition is violated for any $m$ the state is not classical. The bound becomes an equality when the measured state is the coherent state $\left|\alpha_{m, \max }\right\rangle$ with $Q_{m, \max }=Q_{m}\left(\alpha_{m, \max }\right)$. 


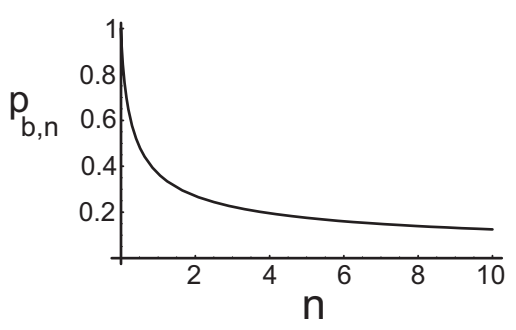

FIG. 1. Classical upper bound [Eq. (3.5)] for the probability of detecting $n$ photons on a classical state.

Moreover, when the measured state is pure $\rho=|\psi\rangle\langle\psi|$ we have that $|\psi\rangle$ is nonclassical if and only if there is at least a measurement for which the classical bound [Eq. (3.2)] is violated. The violation of bound (3.2) is clearly a sufficient condition. This is also necessary since for every nonclassical $|\psi\rangle$ we can consider a POVM with $\Delta_{0}=|\psi\rangle\langle\psi|$. In such a case the upper bound [Eq. (3.2)] is surpassed because $p_{0}=1$ while $\pi Q_{0, \max }=\left|\left\langle\alpha_{\max } \mid \psi\right\rangle\right|^{2}<1$, since otherwise the equality $\left|\left\langle\alpha_{\max } \mid \psi\right\rangle\right|=1$ would imply that $|\psi\rangle$ is a coherent state and thus classical.

Note that the POVM $\Delta_{\alpha}=|\alpha\rangle\langle\alpha| / \pi$ defined by the coherent states $|\alpha\rangle$ (implemented in practice by double homodyne and heterodyne detection) are useless for the detection of nonclassical states since $\pi Q_{\alpha, \max }=1$ and bound (3.2) becomes trivial $p_{\alpha} \leq 1$.

For the sake of illustration we particularize this approach to two meaningful practical situations. These are photonnumber (Sec. III A) and quadrature measurements (Sec. III B). Then we apply them to different measured states (Sec. III C).

\section{A. Photon-number measurement}

For photon-number measurements $\Delta_{n}=|n\rangle\langle n|$ the $Q$ function is

$$
Q_{n}(\alpha)=\frac{1}{\pi} \exp \left(-|\alpha|^{2}\right) \frac{|\alpha|^{2 n}}{n !}
$$

and the maximum occurs at $|\alpha|=\sqrt{n}$;

$$
Q_{n, \max }=\frac{1}{\pi} \exp (-n) \frac{n^{n}}{n !}
$$

If the measured state is classical, the photon-number statistics $p_{n}$ is thus bounded by

$$
p_{n} \leq \exp (-n) \frac{n^{n}}{n !}=p_{b, n}
$$

This bound was previously derived in Ref. [2]. The upper bound $p_{b, n}$ is the probability of detecting $n$ photons in the coherent state $|\alpha\rangle$ with $|\alpha|=\sqrt{n}$, which is the classical state for which $p_{n}$ is maximum. In Fig. 1 we have represented $p_{b, n}$ as a function of $n$ showing that for large $n$ it decays as $p_{b, n}$ $\simeq 1 / \sqrt{2 \pi n}$ approximately, in agreement with the Stirling approximation $n ! \simeq \sqrt{2 \pi n} n^{n} \exp (-n)$.

\section{Independence of sub-Poissonian statistics}

We can show that the nonclassical criterion on photonnumber measurements [Eq. (3.5)] is independent of subPoissonian statistics. The deviation from Poissonian statistics is usually assessed by the Mandel parameter [1]

$$
Q_{M}=\frac{(\Delta n)^{2}}{\langle n\rangle}-1 \text {. }
$$

The independence holds because (i) there are subPoissonian states that satisfy the classical bounds [Eq. (3.5)] for all $n$ and (ii) there are super-Poissonian states that infringe them. To show this let us consider the state in the number basis,

$$
\rho=(1-p)|0\rangle\langle 0|+p| N\rangle\langle N|,
$$

with $0<p \leq 1$ so that

$$
\langle n\rangle=p N, \quad(\Delta n)^{2}=N^{2} p(1-p),
$$

and

$$
Q_{M}=N(1-p)-1 \text {. }
$$

(i) For $N=1$ this state is sub-Poissonian for all $p$ with $Q_{M}$ $=-p<0$ and satisfies the classical upper bounds [Eq. (3.5)] for all $n$ when $p \leq 1 / e$. (ii) For $p<(N-1) / N$ the state is super-Poissonian since $Q_{M}>0$ and infringes bound (3.5) when $p>p_{b, N}$. These two requirements are compatible since it holds that $(N-1) / N>p_{b, N}$ for all $N>1$.

\section{B. Quadrature measurement}

As a further example we may consider the measurement of the quadrature $X$ in Eq. (2.9) so that $\Delta_{x}=|x\rangle\langle x|$. In such a case, from Eq. (2.13) we get $\pi Q_{x, \max }=\sqrt{2 / \pi}$, and the classical upper bound for the statistics $p_{x}$ of the quadrature measurement is

$$
p_{x} \leq \sqrt{\frac{2}{\pi}}=p_{b}
$$

that does not depend on the output $x$. The maximum is obtained for a coherent state $|\alpha\rangle$ with $\left(\alpha+\alpha^{*}\right) / 2=x$. For states with Gaussian $p_{x}$ the infringement of Eq. (3.10) is equivalent to squeezing of quadrature $X$ since the maximum of $p_{x}$ is $1 /(\Delta X \sqrt{2 \pi})$ and

$$
\frac{1}{\Delta X \sqrt{2 \pi}}>\sqrt{\frac{2}{\pi}} \rightarrow \Delta X<\frac{1}{2}=\Delta X_{\text {vacuum }}
$$

For non-Gaussian $p_{x}$ the situation can be different as shown below.

This example is interesting since quadrature measurements are more experimentally feasible than number measurements. For a further discussion about the quantumclassical relation in terms of quadrature distributions see Ref. [11].

\section{Examples}

Let us consider some meaningful simple examples of states violating the classical upper bounds [Eq. (3.5) and (3.10)]. 


\section{Incoherent superposition of thermal and number}

As a feasible state that can infringe Eq. (3.5) let us consider the incoherent superposition of the thermal-chaotic state in Eq. (2.15) and the photon-number state $\left|n_{0}\right\rangle$

$$
\rho=p \rho_{\mathrm{tc}}+(1-p)\left|n_{0}\right\rangle\left\langle n_{0}\right|,
$$

where $0 \leq p \leq 1$, leading to a photon-number statistics

$$
p_{n}=p(1-\xi) \xi^{n}+(1-p) \delta_{n, n_{0}} .
$$

For example, for $p=0.5, n_{0}=1$, and $n_{t c}=9$ the probability of detecting a single photon is $p_{1}=0.545$, while the upper bound in Eq. (3.5) for $n=1$ is $p_{b, 1}=1 / e=0.368$, so we have a clear infringement of the classical condition [Eq. (3.5)] by $100\left(p_{1}-p_{b, 1}\right) / p_{b, 1}=48 \%$.

In this case the photon-number distribution [Eq. (3.13)] is highly super-Poissonian with $Q_{M}=11.2$. Furthermore, we can easily show that there is no quadrature squeezing since for quadrature operators,

$$
X_{\theta}=\frac{1}{2}\left[a^{\dagger} \exp (-i \theta)+a \exp (i \theta)\right],
$$

we have in state (3.12) that $\left\langle X_{\theta}\right\rangle=0$ and

$$
\left(\Delta X_{\theta}\right)^{2}=\frac{1}{2}\langle n\rangle+\frac{1}{4}=\frac{1}{2}\left[p n_{\mathrm{tc}}+(1-p) n_{0}\right]+\frac{1}{4} .
$$

For the above parameters, $n_{0}=1, p=0.5$, and $n_{\mathrm{tc}}=9$, we get $\left(\Delta X_{\theta}\right)^{2}=11 / 4$ for all $\theta$, which is far above the upper limit for squeezing $\left(\Delta X_{\theta}\right)_{\text {vacuum }}^{2}=1 / 4$. Finally, it can be appreciated that there are no oscillations in the photon-number distribution.

For state (3.12) the origin of nonclassical behavior is that $P(\alpha)$ is always more singular than a delta function for all $p \neq 1$. This is because

$$
P(\alpha)=p P_{\mathrm{tc}}(\alpha)+(1-p) P_{n_{0}}(\alpha),
$$

where

$$
P_{\mathrm{tc}}(\alpha)=\frac{1}{\pi n_{\mathrm{tc}}} \exp \left(-\frac{|\alpha|^{2}}{n_{\mathrm{tc}}}\right)
$$

and

$$
P_{n_{0}=1}(\alpha)=\left(1+\frac{\partial}{\partial \alpha} \frac{\partial}{\partial \alpha^{*}}\right) \delta^{(2)}(\alpha) .
$$

Thus $P(\alpha)$ is more singular than a delta function since otherwise we would be able to express $P_{n_{0}=1}(\alpha)$ as a linear combination of two ordinary functions.

\section{Photon-added thermal state}

The previous states are associated with $P$ representatives more singular than a delta function. Next we consider states with nonsingular $P(\alpha)$ function taking negative values. This is the case of the single-photon-added thermal states that, in the photon-number basis, read as [5]

$$
\rho_{1}=(1-\xi) a^{\dagger} \rho_{t c} a=(1-\xi)^{2} \sum_{n=1}^{\infty} \xi^{n-1} n|n\rangle\langle n|,
$$

where $\rho_{\mathrm{tc}}, \xi$, and $n_{\mathrm{tc}}$ are in Eqs. (2.15) and (2.16), respectively.

The $P$ representative is well behaved but nonpositive,

$$
P(\alpha)=\frac{1}{\pi n_{\mathrm{tc}}^{3}}\left[\left(n_{\mathrm{tc}}+1\right)|\alpha|^{2}-n_{\mathrm{tc}}\right] \exp \left(-|\alpha|^{2} / n_{\mathrm{tc}}\right),
$$

and the photon-number statistics is

$$
p_{n}=(1-\xi)^{2} \xi^{n-1} n
$$

For example, for $n=1,2$ the classical upper bounds [Eq. (3.5)] are surpassed provided that

$$
\begin{aligned}
& p_{1}>p_{b, 1}=1 / e \leftrightarrow n_{\mathrm{tc}}<\sqrt{e}-1=0.65, \\
& p_{2}>p_{b, 2}=2 / e^{2} \leftrightarrow 0.30 \leq n_{\mathrm{tc}} \leq 0.82 .
\end{aligned}
$$

Let us show that this nonclassical behavior is independent of other nonclassical features. There is no quadrature squeezing since for the rotated quadrature operators $X_{\theta}$ in Eq. (3.14) we have $\left(\Delta X_{\theta}\right)^{2}=\left(3+4 n_{\mathrm{tc}}\right) / 4>1 / 4$ for all $\theta$. Also, it can be appreciated in Eq. (3.21) that there is no photon-number oscillations. Finally, the Mandel parameter is

$$
Q_{M}=\frac{2 n_{\mathrm{tc}}^{2}-1}{2 n_{\mathrm{tc}}+1}
$$

so we get super-Poissonian statistics for all $n_{\mathrm{tc}}>1 / \sqrt{2}$ $=0.71$.

Therefore, the states with $0.71 \leq n_{\mathrm{tc}} \leq 0.82$ are nonclassical since $p_{2}>p_{b, 2}$, although they have super-Poissonian statistics, present no squeezing, and have no oscillatory statistics.

\section{Coherent superposition of coherent states}

Another interesting example is provided by the coherent superposition of two coherent states with opposed complex amplitude [12] (referred to as even and odd superpositions [13])

$$
\left|\alpha_{ \pm}\right\rangle=N_{ \pm}(|\alpha\rangle \pm|-\alpha\rangle),
$$

with

$$
N_{+}=\frac{\exp \left(|\alpha|^{2} / 2\right)}{2 \sqrt{\cosh \left(|\alpha|^{2}\right)}}, \quad N_{-}=\frac{\exp \left(|\alpha|^{2} / 2\right)}{2 \sqrt{\sinh \left(|\alpha|^{2}\right)}}
$$

In this case the $P(\alpha)$ is a distribution involving an infinite number of derivatives of the delta function since the normally ordered characteristic function is a real exponential. For definiteness let us focus just on the even states $\left|\alpha_{+}\right\rangle$.

For the even case $\left|\alpha_{+}\right\rangle$we have the following photonnumber statistics: 


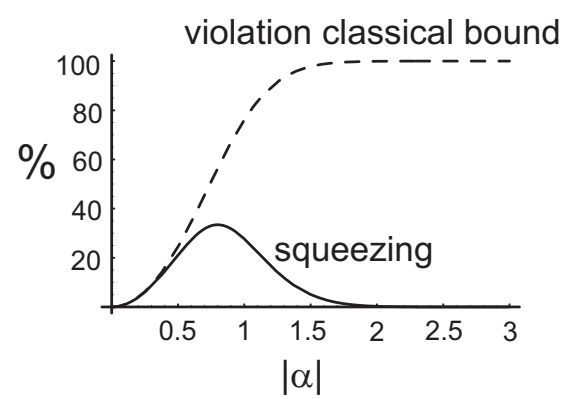

FIG. 2. Percentage $100\left(p_{0}-p_{b}\right) / p_{b}$ of maximum violation of the classical bound [Eq. (3.10)] (dashed line) and percentage of squeezing $100\left(1-2 \Delta X_{\theta, \text { min }}\right)$ in Eq. (3.32) (solid line) for the even state (3.24) as functions of $|\alpha|$.

$$
p_{n}=\left\{\begin{array}{cc}
\frac{|\alpha|^{2 n}}{n ! \cosh \left(|\alpha|^{2}\right)} & \text { for } n \text { even } \\
0 & \text { for } n \text { odd }
\end{array}\right.
$$

Numerically it can be easily seen that this is inconsistent with the upper bound in Eq. (3.5) for $|\alpha| \geq 0.64$. Moreover, for $|\alpha| \gg 1$ we have $\cosh \left(|\alpha|^{2}\right) \simeq \exp \left(|\alpha|^{2}\right) / 2$ and (always for even $n$ )

$$
p_{n} \simeq 2 \frac{|\alpha|^{2 n}}{n !} \exp \left(-|\alpha|^{2}\right)
$$

so that for $|\alpha|^{2}=n$ we get that $p_{n}$ is twice the upper bound $p_{b, n}$ in Eq. (3.5).

Concerning quadrature measurements, let us consider states with purely imaginary complex amplitude $\alpha= \pm i|\alpha|$ that have the following quadrature statistics:

$$
p_{x}=4 N_{+}^{2} \sqrt{\frac{2}{\pi}} \cos ^{2}(2|\alpha| x) \exp \left(-2 x^{2}\right) .
$$

For every $|\alpha|$ the maximum of $p_{x}$ holds for $x=0$ being $p_{0}$ $=4 N_{+}^{2} \sqrt{2 / \pi}$. In Fig. 2 (dashed line) we have represented the relative amount of violation of Eq. (3.10), $100\left(p_{0}-p_{b}\right) / p_{b}$, as a function of $|\alpha|$, showing a $100 \%$ violation for large $|\alpha|$. This is because for $|\alpha| \gg 1$ we have $N_{+}^{2} \simeq 1 / 2$ so that $p_{0}$ $\simeq 2 \sqrt{2 / \pi}$, which is twice the classical upper bound $p_{b}$ $=\sqrt{2 / \pi}$. In Fig. 2 this is also compared with the percentage of squeezing in the same state (solid line).

Next we show that the even states infringe classical bounds with super-Poissonian photon-number statistics and with negligible quadrature squeezing. Concerning photonnumber statistics we have

$$
\begin{gathered}
\langle n\rangle=|\alpha|^{2} \tanh \left(|\alpha|^{2}\right), \\
\left\langle n^{2}\right\rangle=|\alpha|^{4}+|\alpha|^{2} \tanh \left(|\alpha|^{2}\right)
\end{gathered}
$$

so that

$$
Q_{M}=\frac{2|\alpha|^{2}}{\sinh \left(2|\alpha|^{2}\right)},
$$

and these states are always super-Poissonian (unless $\alpha=0$ ).
Concerning quadrature squeezing, the minimum uncertainty for rotated quadratures [Eq. (3.14)] in the state $\left|\alpha_{+}\right\rangle$ when $\theta$ is varied is

$$
\left(\Delta X_{\theta}\right)_{\min }^{2}=\frac{1}{4}\left(2|\alpha|^{2} \tanh \left(|\alpha|^{2}\right)-2|\alpha|^{2}+1\right) .
$$

The percentage of squeezing defined as

$$
100 \frac{\Delta X_{\text {vacuum }}-\Delta X_{\theta, \text { min }}}{\Delta X_{\text {vacuum }}}=100\left(1-2 \Delta X_{\theta, \text { min }}\right)
$$

is represented in Fig. 2 as a function of $|\alpha|$. These states present squeezing only for small $|\alpha|$ being negligible for $|\alpha|>2$. For instance, for $|\alpha|=3$ we have $\left(\Delta X_{\theta, \min }\right)^{2}$ $=0.24999986$, which means a fully negligible 2.75 $\times 10^{-5} \%$ squeezing, while Eqs. (3.5) and (3.10) are infringed by a $100 \%$ for the same state.

At difference with the preceding examples in this case the $100 \%$ violation of classical bounds for large $|\alpha|$ has a simple explanation in terms of the oscillatory character of the statistics [Eqs. (3.26) and (3.28)]. For large $|\alpha|$ the number and quadrature statistics are the same of coherent states (that would saturate the classical bounds) but maximally modulated. Because of normalization, the vanishing terms must be compensated by nonvanishing terms reaching twice the coherent-state values. This factor of 2 leads to the $100 \%$ violation of the classical bounds.

Let us note that the criteria presented in this work reveal the nonclassical nature of these states for all $\alpha$ but specially clearly for large $|\alpha|$. This is sharp contrast with subPoissonian number statistics and quadrature squeezing that hold only for small $|\alpha|$, as illustrated in Fig. 2 for example.

\section{EFFECT OF IMPERFECTIONS}

One of the key features of this approach is that the data analysis is reduced to minimum. This favors obtaining reliable results from nonideal measurements affected by imperfections, such as damping, finite efficiencies, or finite sampling. We stress that this approach applies to any measurement, both ideal and imperfect, so that experimental imperfections can be always embodied into the measuring POVM. Nevertheless, since imperfections usually deteriorate nonclassical properties it is reasonable to investigate their effect on the above nonclassical criteria.

\section{A. Inefficient detection}

For definiteness we consider real detectors affected by field damping (with bath at zero temperature) and finite quantum efficiency, which can be modeled by placing a beam splitter of amplitude-transmission coefficient $t=\sqrt{\eta}$ in front of a perfect detector, where $\eta \leq 1$ represents both losses and efficiencies $[14,15]$.

\section{Nonclassical states}

The effect of the beam splitter for the detection of nonclassical states can be easily accounted for by computing the measured state after the beam splitter $\rho_{t}$ as 


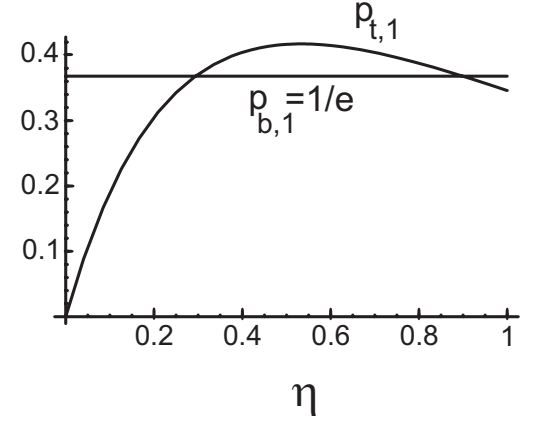

FIG. 3. $p_{t, 1}$ in Eq. (4.3) as a function of $\eta$ for $n_{\mathrm{tc}}=0.7$.

$$
\rho_{t}=\int d^{2} \alpha P(\alpha)|t \alpha\rangle\langle t \alpha|,
$$

where $P(\alpha)$ is the $P$ function of the measured state. The measured statistics becomes

$$
p_{t, m}=\operatorname{tr}\left(\Delta_{m} \rho_{t}\right)=\pi \int d^{2} \alpha P(\alpha) Q_{m}(t \alpha) .
$$

Since the maximum of $Q_{m}(t \alpha)$ when $\alpha$ is varied is the same as the maximum of $Q_{m}(\alpha)$, there is no change in the classical upper bound in the right-hand side of Eq. (3.2).

Nevertheless, imperfections affect the statistics replacing $p_{m}$ by $p_{t, m}$ in the left-hand side of Eq. (3.2). This can be easily seen, for example, for photon-added thermal states [Eq. (3.19)]. For inefficient detection the photon-number statistics of ideal case (3.21) for $n=1$ is replaced by

$$
p_{t, 1}=\eta \frac{1+2 n_{\mathrm{tc}}-\eta n_{\mathrm{tc}}}{\left(1+\eta n_{\mathrm{tc}}\right)^{3}} .
$$

In Fig. 3 we have represented $p_{t, 1}$ as a function of $\eta$ for $n_{\mathrm{tc}}$ $=0.7$. Decreasing $\eta$ from $\eta=1$ increases $p_{t, 1}$, leading to break the classical bound [Eq. (3.5)] in the interval $0.30 \leq \eta$ $\leq 0.89$.

It is worth pointing out that, at difference with other nonclassical tests, where imperfections degrade nonclassical behavior [15], in this case larger losses and decreasing efficiencies may favor the observation of nonclassical behavior of measured states. This noticeable effect arises because imperfections rearrange the probability distribution $p_{n}$ so that with increasing imperfection some probabilities may increase beyond the classical bounds, as is the case of $p_{t, 1}$ in this example.

\section{Nonclassical measurements}

For nonclassical measurements we can follow two different strategies: (i) we can address the nonclassical behavior of the ideal POVM $\Delta_{m}$ associated to $\eta=1$. This is the analog of the preceding subsection where we investigated the nonclassical properties of the input state before being affected by imperfections. (ii) Alternatively, as mentioned above we can examine the nonclassical behavior of the effective POVM $\widetilde{\Delta}_{m}$ embodying all imperfections as part of the measuring scheme. (i) Concerning the nonclassical behavior of the ideal $\operatorname{POVM} \Delta_{m}$ we can compute the effect of inefficiencies as

$$
p_{t, m}=\frac{1}{\pi} \int d^{2} \alpha d^{2} \beta_{s}\left\langle\left.\alpha\right|_{a}\left\langle\beta\left|T \rho \otimes \rho_{0} T^{\dagger}\right| \beta\right\rangle_{a} \mid \alpha\right\rangle_{s} P_{m}(\alpha),
$$

where $|\alpha\rangle_{s}$ are coherent states in the signal mode (the mode of the measured state $\rho$ ) and $|\beta\rangle_{a}$ are coherent states in the auxiliary mode (the other input port of the beam splitter assumed in the vacuum state $\left.\rho_{0}\right), P_{m}(\alpha)$ is the $P$ representative of the ideal measurement $\Delta_{m}$, and $T$ is the unitary transformation describing the effect of the beam splitter, with

$$
T^{\dagger}|\beta\rangle_{a}|\alpha\rangle_{s}=|t \beta+r \alpha\rangle_{a}|t \alpha-r \beta\rangle_{s},
$$

being $r=\sqrt{1-t^{2}}$. This leads to the following form for the statistics

$$
p_{t, m}=\pi \int d^{2} \alpha \widetilde{Q}(\alpha) P_{m}(\alpha)
$$

where $\widetilde{Q}(\alpha)$ is defined here as

$$
\widetilde{Q}(\alpha)=\int d^{2} \beta Q_{0}(t \beta+r \alpha) Q(t \alpha-r \beta),
$$

with $Q_{0}$ and $Q$ being the $Q$ representatives of $\rho_{0}$ and $\rho$, respectively. Note that in the ideal case $\eta=1 \quad(t=1, r=0)$ $\widetilde{Q}(\alpha)$ is the $Q$ function of the measured state $\widetilde{Q}(\alpha)=Q(\alpha)$. From Eq. (4.6) we can derive the classical upper bound

$$
p_{t, m} \leq \pi \widetilde{Q}_{\max } \operatorname{tr} \Delta_{m}
$$

which holds for classical measurements with $P_{m}(\alpha) \geq 0$. We can appreciate that finite quantum efficiencies modify the classical upper bounds in comparison with the ideal detection in Eq. (2.2) by replacing $Q_{\max }$ by $\widetilde{Q}_{\max }$.

Let us illustrate this analysis with the example where the ideal POVM is one-photon detection, $\Delta_{1}=|1\rangle\langle 1|$ in the number basis, and the measured state is the one-photon state $|1\rangle$. The case $\eta=1$ was considered in Sec. II A above. When $\eta$ $\leq 1$ we have

$$
p_{t, 1}=\eta, \quad \operatorname{tr} \Delta_{1}=1,
$$

and

$$
\widetilde{Q}(\alpha)=\frac{1}{\pi}\left[\eta\left(|\alpha|^{2}-1\right)+1\right] \exp \left(-|\alpha|^{2}\right)
$$

so that

$$
\widetilde{Q}_{\max }=\frac{\eta}{\pi} \exp \left(-\frac{2 \eta-1}{\eta}\right)
$$

and the violation of the classical upper bound [Eq. (4.8)] occurs provided that 


$$
\exp \left(-\frac{2 \eta-1}{\eta}\right)<1 \leftrightarrow \eta>\frac{1}{2} .
$$

Therefore, in this example the nonclassical behavior of the ideal measurement is disclosed provided that the quantum efficiencies are above $50 \%$.

(ii) Alternatively, if we embody decaying mechanisms and inefficiencies in the effective POVM $\widetilde{\Delta}_{m}$ we get from Eq. (2.2)

$$
p_{t, m} \leq \pi Q_{\max } \operatorname{tr} \tilde{\Delta}_{m}
$$

where $Q_{\max }$ is the maximum of the $Q$ function of the measured state $\rho$. We can compute $\operatorname{tr} \widetilde{\Delta}_{m}$ taking into account the effect of the beam splitter as in Eq. (4.1);

$$
\operatorname{tr} \widetilde{\Delta}_{m}=\frac{1}{\pi} \int d^{2} \alpha\left\langle\alpha\left|\widetilde{\Delta}_{m}\right| \alpha\right\rangle=\frac{1}{\pi} \int d^{2} \alpha\left\langle t \alpha\left|\Delta_{m}\right| t \alpha\right\rangle=\frac{1}{t^{2}} \operatorname{tr} \Delta_{m}
$$

leading to

$$
p_{t, m} \leq \pi Q_{\max } \frac{1}{\eta} \operatorname{tr} \Delta_{m} .
$$

We can appreciate that the effect of imperfections is simply expressed by increasing the classical upper bound by a factor of $1 / \eta$.

Let us illustrate this approach with the same example of inefficient one-photon detection, (ideal POVM $\Delta_{1}=|1\rangle\langle 1|$ and a one-photon state $|1\rangle)$ so that after Eq. (2.7) the classical bound [Eq. (4.15)] is

$$
p_{t, 1}=\eta \leq \frac{1}{\eta e} .
$$

Thus the effective POVM $\widetilde{\Delta}_{m}$ shows nonclassical behavior when $\eta>1 / \sqrt{e}=0.6065$.

We can appreciate that the two approaches (i) and (ii) lead to two different bounds, Eqs. (4.8) and (4.15), as clearly illustrated by the example of one-photon detection. We stress that this difference is natural since the classical bound [Eq. (4.8)] is sensitive to the nonclassicality of the $P$ representative of the ideal POVM $\Delta_{m}$, while bound (4.15) is sensitive to the nonclassical character of the $P$ representative of the effective POVM $\widetilde{\Delta}_{m}$.

\section{B. Finite sampling}

When the number of measurements $N$ is finite, the probability $p_{m}$ becomes an statistical variable that can be expressed as $p_{m}(N)=k / N$, where the integer $k$ is the number of outcomes $m$ after $N$ trials. (This analysis applies both to detection of nonclassical states and measurements.) The dichotomic character of the measurement (outcome $m$ with probability $p_{m}$ and outcome not $m$ with probability $1-p_{m}$ ) implies that $k$ follows the binomial distribution

$$
\mathcal{P}_{k}(N)=\left(\begin{array}{l}
N \\
k
\end{array}\right) p_{m}^{k}\left(1-p_{m}\right)^{N-k},
$$

so we have

$$
\left\langle p_{m}(N)\right\rangle=\frac{\langle k\rangle}{N}=p_{m}, \quad \Delta p_{m}(N)=\frac{\Delta k}{N}=\sqrt{\frac{p_{m}\left(1-p_{m}\right)}{N}} .
$$

For all the above examples we have roughly $p_{m} \simeq 0.5$ so that for $N \simeq 100$ we have $\Delta p_{m} / p_{m} \simeq 0.1$. Thus, even for moderate number of trials, the uncertainty caused by finite sampling is clearly below the amount of violation of classical upper bounds $p_{b, m}$ since $\left(p_{m}-p_{b, m}\right) / p_{b, m}$ is at least five times larger than $\Delta p_{m} / p_{m}$ in the above examples.

\section{TWO-MODE OBSERVABLES}

The above single-mode approach in Eq. (1.3) can be easily generalized to two-mode observables by expressing the statistics $p_{m}=\operatorname{tr}\left(\rho \Delta_{m}\right)$ as

$$
p_{m}=\pi^{2} \int d^{2} \alpha d^{2} \beta P(\alpha, \beta) Q_{m}(\alpha, \beta)
$$

and

$$
p_{m}=\pi^{2} \int d^{2} \alpha d^{2} \beta P_{m}(\alpha, \beta) Q(\alpha, \beta),
$$

where $P$ and $Q$ are the two-mode phase-space representatives for the measured state $\rho$;

$$
\begin{gathered}
\rho=\int d^{2} \alpha d^{2} \beta P(\alpha, \beta)|\alpha, \beta\rangle\langle\alpha, \beta|, \\
Q(\alpha, \beta)=\frac{1}{\pi^{2}}\langle\alpha, \beta|\rho| \alpha, \beta\rangle,
\end{gathered}
$$

where $P_{m}$ and $Q_{m}$ refer to the corresponding representatives of the POVM $\Delta_{m}$ and $|\alpha, \beta\rangle$ are two-mode coherent states. The parameter $m$ represents all the indices necessary to label the outcomes. The maxima of $Q(\alpha, \beta)$ and $Q_{m}(\alpha, \beta)$ provide suitable upper bounds for the statistics of classical measurements and states, respectively.

In this regard we note that for bipartite systems nonclassical $P(\alpha, \beta)$ is a necessary condition for entanglement [16]. For definiteness we focus on the nonclassical behavior of states. The analysis of nonclassical measurements would be analogous.

\section{A. Nonclassical states by photon-number detection}

For the case of joint two-mode photon-number detection we get that for classical states the joint probability of detecting $n_{1}$ and $n_{2}$ photons is simply bounded by the product of the one-mode upper bounds

$$
p_{n_{1}, n_{2}} \leq p_{b, n_{1}} p_{b, n_{2}}=\exp \left[-\left(n_{1}+n_{2}\right)\right] \frac{n_{1}^{n_{1}} n_{2}^{n_{2}}}{n_{1} ! n_{2} !} \text {. }
$$

The maximum for fixed $n_{1}+n_{2}$ occurs when $n_{1}=0$ or $n_{2}=0$, while the minimum occurs for coincident outputs $n_{1}=n_{2}$.

For the total number $n=n_{1}+n_{2}$ the statistics is given by 


$$
p_{n}=\pi^{2} \int d^{2} \alpha d^{2} \beta P(\alpha, \beta) \sum_{m=0}^{n} Q_{m, n-m}(\alpha, \beta),
$$

where

$$
Q_{n_{1}, n_{2}}(\alpha, \beta)=\frac{1}{\pi^{2}}\left|\left\langle\alpha \mid n_{1}\right\rangle\right|^{2}\left|\left\langle\beta \mid n_{2}\right\rangle\right|^{2},
$$

$\left|n_{1,2}\right\rangle$ being number states in the corresponding modes. It can be easily seen that

$$
\sum_{m=0}^{n} Q_{m, n-m}(\alpha, \beta)=\frac{1}{\pi^{2}} \frac{|\gamma|^{2 n}}{n !} \exp \left(-|\gamma|^{2}\right),
$$

with $|\gamma|^{2}=|\alpha|^{2}+|\beta|^{2}$. For fixed $n$ the maximum occurs for $|\gamma|^{2}=n$ so that

$$
\sum_{m=0}^{n} Q_{m, n-m}(\alpha, \beta) \leq \frac{1}{\pi^{2}} \frac{n^{n}}{n !} \exp (-n)
$$

and

$$
p_{n} \leq \frac{n^{n}}{n !} \exp (-n)
$$

which is equal to the single-mode counterpart [Eq. (3.5)].

\section{B. Nonclassical states by quadrature-difference measurement}

Let us consider the measurement of the quadrature difference $X=X_{1}-X_{2}$, where $X_{1,2}$ represent the same quadrature operator in each mode, which is described by the POVM

$$
\Delta_{x}=\int d x^{\prime}\left|x+x^{\prime}\right\rangle_{1}\left\langle x+x^{\prime}|\otimes| x^{\prime}\right\rangle_{2}\left\langle x^{\prime}\right|,
$$

where $|x\rangle_{j}$ are the eigenstates of $X_{j}$, with $j=1,2$. In this case we have

$$
Q_{x}\left(\alpha_{1}, \alpha_{2}\right)=\frac{1}{\pi^{2} \sqrt{\pi}} \exp \left[-\left(x-x_{1}+x_{2}\right)^{2}\right],
$$

where $x_{j}$ is the real part of $\alpha_{j}$ so that

$$
\pi^{2} Q_{x, \max }=\frac{1}{\sqrt{\pi}},
$$

and the classical bound is

$$
p_{x} \leq \frac{1}{\sqrt{\pi}}=p_{b}
$$

Note that the two-mode bound does not depend on the outcome $x$, being lower than the single-mode counterpart [Eq. (3.10)]. For states with Gaussian $p_{x}$ the violation of this bound is equivalent to $\Delta X<1 / \sqrt{2}$. This is equivalent to twomode squeezing since for pairs of coherent states it holds $\Delta X=1 / \sqrt{2}$.

\section{Example: Two-mode squeezed vacuum}

To illustrate these two-mode classical bounds let us consider that the measured state is a two-mode squeezed vacuum that in the photon-number basis reads as

$$
|\zeta\rangle=\sqrt{1-\zeta^{2}} \sum_{n=0}^{\infty} \zeta^{n}|n\rangle_{1}|n\rangle_{2},
$$

where we have assumed real parameter $\zeta$ without loss of generality. The statistic of the joint number $p_{n, n}$ and the total number $p_{2 n}$ are

$$
p_{n, n}=p_{2 n}=\left(1-\zeta^{2}\right) \zeta^{2 n}
$$

while the statistics of the quadrature-difference $X=X_{1}-X_{2}$ is Gaussian with

$$
(\Delta X)^{2}=\frac{1-\zeta}{2(1+\zeta)} .
$$

Numerically we have found that the classical bound on joint-number measurements is always violated $p_{n, n}>p_{b, n}^{2}$ for some $n$ when $\zeta>0.41$. The classical bound for total number is never violated since $p_{2 n}=p_{n, n} \leq p_{b, 2 n}$ for all $\zeta$.

More specifically, for $n=1$ we have $p_{1,1}=\left(1-\zeta^{2}\right) \zeta^{2}>p_{b, 1}^{2}$ $=\exp (-2)=0.135$ for all $\zeta$ in the interval $0.41 \leq \zeta \leq 0.91$. The maximum violation occurs for $\zeta^{2}=1 / 2$ so that $p_{1,1}=0.25$, and there is an $85 \%$ violation of the classical bound. For the total number we have that the classical bound is not surpassed since $p_{b, 2}=2 \exp (-2)=0.27$.

On the other hand the classical bound on quadrature difference is always surpassed since the statistics is Gaussian and $\Delta X<1 / \sqrt{2}$ for all $\zeta$. For example, for $\zeta^{2}=1 / 2$ (this is mean total number of photons $\langle n\rangle=2$ ) we have $p_{0}=1.36$, while the classical upper bound in Eq. (5.14) is $p_{b}=0.56$ so that there is a percentage of violation $100\left(p_{0}-p_{b}\right) / p_{b}$ $=141 \%$ approximately. The outputs $x$ that contravene Eq. (5.14) are all $x$ in the interval $-0.39 \leq x \leq 0.39$, which represent an $82 \%$ probability since $\int_{-0.39}^{0.39} p_{x} d x=0.82$.

\section{SPIN SYSTEMS}

The above methods can be adapted to situations described by finite-dimensional Hilbert spaces, exemplified by spin- $j$ systems. This can be readily done in terms of $\mathrm{SU}(2) Q$ and $P$ functions, which are defined after the $\mathrm{SU}(2)$ coherent states $|j, \Omega\rangle$ as [17]

$$
\rho=\int d^{2} \Omega P(\Omega)|j, \Omega\rangle\langle j, \Omega|, \quad Q(\Omega)=\frac{2 j+1}{4 \pi}\langle j, \Omega|\rho| j, \Omega\rangle,
$$

with $d^{2} \Omega=\sin \theta d \theta d \phi$, and

$$
\begin{aligned}
|j, \Omega\rangle= & \sum_{m=-j}^{j}\left(\begin{array}{c}
2 j \\
m+j
\end{array}\right)^{1 / 2} \sin ^{j-m}\left(\frac{\theta}{2}\right) \cos ^{j+m}\left(\frac{\theta}{2}\right) \\
& \times \exp [-i(j+m) \phi]|j, m\rangle,
\end{aligned}
$$

where $|j, m\rangle$ are the eigenstates of the spin component $j_{3}$ with eigenvalue $m$, while $\pi \geq \theta \geq 0$ and $\pi \geq \phi \geq-\pi$. The analog of Eq. (1.3) is 


$$
p_{m}=\frac{4 \pi}{2 j+1} \int d^{2} \Omega P_{m}(\Omega) Q(\Omega)=\frac{4 \pi}{2 j+1} \int d^{2} \Omega P(\Omega) Q_{m}(\Omega),
$$

leading to the following bounds for classical measurements

$$
p_{m} \leq \frac{4 \pi}{2 j+1} Q_{\max } \operatorname{tr} \Delta_{m}
$$

(for finite-dimensional systems tr $\Delta_{m}$ is always finite), while the upper bound for classical states is

$$
p_{m} \leq \frac{4 \pi}{2 j+1} Q_{m, \max }=p_{b, m} .
$$

By construction the SU(2) coherent states are classical both as measured and measuring states. For the POVM,

$$
\Delta_{\Omega}=\frac{2 j+1}{4 \pi}|j, \Omega\rangle\langle j, \Omega|, \quad \operatorname{tr} \Delta_{\Omega}=\frac{2 j+1}{4 \pi},
$$

the upper bound [Eq. (6.4)] is satisfied for all measured states since

$$
p_{\Omega}=Q(\Omega) \leq \frac{4 \pi}{2 j+1} Q_{\max } \operatorname{tr} \Delta_{\Omega}=Q_{\max },
$$

where $Q(\Omega)$ is the $\mathrm{SU}(2) Q$ function of the measured state. Likewise, when the measured state is coherent $\rho$ $=|j, \Omega\rangle\langle j, \Omega|$ we have

$$
p_{m}=\frac{4 \pi}{2 j+1} Q_{m}(\Omega),
$$

where $Q_{m}(\Omega)$ is the $\mathrm{SU}(2) Q$ function of $\Delta_{m}$, so that Eq. (6.5) is satisfied by all measurements.

For the sake of illustration next we consider some examples for the simplest cases $j=1 / 2$ and $j=1$.

$$
\text { A. } j=1 / 2
$$

For $j=1 / 2$ the $\mathrm{SU}(2)$ coherent states read, in the $|j, m\rangle$ basis, as

$$
|1 / 2, \Omega\rangle=\sin \left(\frac{\theta}{2}\right)|1 / 2,-1 / 2\rangle+\cos \left(\frac{\theta}{2}\right) \exp (-i \phi)|1 / 2,1 / 2\rangle .
$$

Every state and POVM are of the form

$$
\begin{gathered}
\rho=\frac{1}{2}\left(\sigma_{0}+\boldsymbol{r} \cdot \boldsymbol{\sigma}\right), \quad Q(\Omega)=\frac{1}{4 \pi}(1+\boldsymbol{r} \cdot \boldsymbol{\Omega}), \\
\Delta_{m}=\lambda_{m}\left(\sigma_{0}+\boldsymbol{r}_{m} \cdot \boldsymbol{\sigma}\right), \quad Q_{m}(\Omega)=\frac{\lambda_{m}}{2 \pi}\left(1+\boldsymbol{r}_{m} \cdot \boldsymbol{\Omega}\right),
\end{gathered}
$$

where $\sigma$ are the Pauli matrices in the $|j, m\rangle$ basis, $\sigma_{0}$ is the identity, $\boldsymbol{r}$ and $\boldsymbol{r}_{m}$ are three-dimensional real vectors with $|\boldsymbol{r}|,\left|\boldsymbol{r}_{m}\right| \leq 1, \boldsymbol{\Omega}=(\sin \theta \cos \phi, \sin \theta \sin \phi, \cos \theta)$, and $\lambda_{m} \geq 0$.

Let us show that for $j=1 / 2$ no state exhibits nonclassical properties. This is because for every $\Delta_{m}$ and using that $\operatorname{tr}\left(\sigma_{j} \sigma_{k}\right)=2 \delta_{j, k}$,

$$
p_{m}=\operatorname{tr}\left(\rho \Delta_{m}\right)=\lambda_{m}\left(1+\boldsymbol{r}_{m} \cdot \boldsymbol{r}\right), \quad Q_{m, \max }=\frac{\lambda_{m}}{2 \pi}\left(1+\left|\boldsymbol{r}_{m}\right|\right)
$$

so that $p_{m}>p_{b, m}$ would imply $\boldsymbol{r}_{m} \cdot \boldsymbol{r}>\left|\boldsymbol{r}_{m}\right|$, which is not possible since $|\boldsymbol{r}| \leq 1$.

Similarly, there are no measurements exceeding the classical bounds since $p_{m}=\lambda_{m}\left(1+\boldsymbol{r}_{m} \cdot \boldsymbol{r}\right), Q_{\max }=(1+|\boldsymbol{r}|) /(4 \pi)$, and $\operatorname{tr} \Delta_{m}=2 \lambda_{m}$ so that the violation of the classical bound [Eq. (6.4)] would be equivalent to $\left.\boldsymbol{r}_{m} \cdot \boldsymbol{r}\right\rangle|\boldsymbol{r}|$, which is not possible since $\left|\boldsymbol{r}_{m}\right| \leq 1$.

This lack of nonclassical states agrees with the approach in Ref. [18] and with the fact that for $j=1 / 2$ all pure states are $\mathrm{SU}(2)$ coherent states. On the other hand, this is in sharp contrast with the fact that all pure state have negative values of the SU(2) Wigner function [19]. Nevertheless, it is worth pointing out that for finite-dimensional systems the $\mathrm{SU}(2)$ distributions such as $P(\Omega)$ and the Wigner function are not uniquely defined in contrast with their infinite-dimensional counterparts $[18,19]$.

$$
\text { B. } j=1
$$

For $j=1$ the coherent states [Eq. (6.2)] are

$$
\begin{aligned}
|1, \Omega\rangle= & \sin ^{2}\left(\frac{\theta}{2}\right)|1,-1\rangle+\sqrt{2} \sin \left(\frac{\theta}{2}\right) \cos \left(\frac{\theta}{2}\right) \exp (-i \phi)|1,0\rangle \\
& +\cos ^{2}\left(\frac{\theta}{2}\right) \exp (-i 2 \phi)|1,1\rangle .
\end{aligned}
$$

\section{Nonclassical states}

As a measurement revealing nonclassical states we can consider the projection on the state $|1,0\rangle$ (in the $|j, m\rangle$ basis),

$$
\Delta_{0}=\rho_{0}=|1,0\rangle\langle 1,0|,
$$

with $Q$ function,

$$
Q_{0}(\Omega)=\frac{3}{8 \pi} \sin ^{2} \theta, \quad Q_{0, \max }=\frac{3}{8 \pi},
$$

so that the classical upper bound in Eq. (6.5) is $p_{b, 0}=1 / 2$. This is clearly violated when the measured state is the same state $\rho=\rho_{0}$ since in such a case $p_{0}=1>p_{b, 0}=1 / 2$, and there is a $100 \%$ violation of the classical bound.

This agrees with the fact that the state $|1,0\rangle$ in Eq. (6.14) can be regarded as the limit of SU(2) squeezed states [20,21]. This also agrees with the result in Ref. [18] stating that for $j=1$ classical behavior [i.e., nonsingular positive $P(\Omega)$ ] is equivalent to non-negative covariancelike matrix

$$
Z_{k, \ell}=\left\langle\left(j_{k} j_{\ell}+j_{\ell} j_{k}\right)\right\rangle-\delta_{k, \ell}-\left\langle j_{k}\right\rangle\left\langle j_{\ell}\right\rangle .
$$

For the state $|1,0\rangle$ in Eq. (6.14) we have [21]

$$
Z=\left(\begin{array}{ccc}
1 & 0 & 0 \\
0 & 1 & 0 \\
0 & 0 & -1
\end{array}\right)
$$

so that the state is nonclassical. 


\section{Nonclassical measurements}

We can provide an example of nonclassical measurement. To this end we consider the measurement (in the $|j, m\rangle$ basis) $\Delta_{0}=\rho_{0}=|1,0\rangle\langle 1,0|$. As measured state we consider the phase averaged equatorial $\mathrm{SU}(2)$ coherent state

$$
\rho=\frac{1}{2 \pi} \int_{2 \pi} d \phi|1, \theta=\pi / 2, \phi\rangle\langle 1, \theta=\pi / 2, \phi|,
$$

where $|j, \theta, \phi\rangle$ are the $\mathrm{SU}(2)$ coherent states. In the $|j, m\rangle\langle j, m|$ basis this is

$$
\rho=\frac{1}{4}|1,1\rangle\left\langle 1,1\left|+\frac{1}{2}\right| 1,0\right\rangle\left\langle 1,0\left|+\frac{1}{4}\right| 1,-1\right\rangle\langle 1,-1|,
$$

with $Q$ function

$$
Q(\Omega)=\frac{3}{16 \pi}\left(1+\frac{1}{2} \sin ^{2} \theta\right), \quad Q_{\max }=\frac{9}{32 \pi} .
$$

The probability is $p_{0}=1 / 2$, which is $167 \%$ above the classical bound in Eq. (6.4),

$$
p_{b}=\frac{4 \pi}{2 j+1} Q_{\max } \operatorname{tr} \Delta_{0}=\frac{3}{8} .
$$

\section{CONCLUSIONS}

We have provided feasible practical procedures to reveal the quantum nature of states and measurements. We have illustrated them with the most practical measuring schemes available, such as photon-number and quadrature measurements.

The nonclassical tests proposed in this approach are exceedingly simple since definite conclusions are obtained without evaluation of moments or any other more sophisticated data analysis. This is reflected on the robustness of these nonclassical criteria under practical imperfections, such as finite detection efficiencies and finite sampling.

We have demonstrated that these nonclassical tests are independent of other typical quantum signatures such as subPoissonian statistics, quadrature squeezing, or oscillatory statistics.

\section{ACKNOWLEDGMENTS}

We thank Dr. Shashank Virmani for fruitful discussions and Professor Mark Hillery for valuable comments. A.R. acknowledges financial support from the University of Hertfordshire and the EU Integrated Project QAP. A.L. acknowledges support from Project No. FIS2008-01267 of the Spanish Dirección General de Investigación del Ministerio de Ciencia e Innovación.

\section{APPENDIX: TRACE MEASUREMENTS}

As we have shown above, the classical bounds for measurements depend on the trace of the corresponding POVM elements $\Delta_{m}$. Incidentally, the trace $\operatorname{tr} \Delta_{m}$ is proportional to the probability of the outcome $m$ when the input state is the one of maximum ignorance, $\rho \propto I$, where $I$ is the identity;

$$
p_{m}(\rho \propto I) \propto \operatorname{tr} \Delta_{m} .
$$

When $\operatorname{tr} \Delta_{m}$ depends on $m$ this can be regarded as a kind of prior bias, since some outcomes are more probable than others even when the measured state presents in principle no preference for any outcome.

In this appendix we present two simple procedures that allow us to determine $\operatorname{tr} \Delta_{m}$ in practice. For simplicity and without loss of generality we focus on the single-mode case.

To this end we note that the identity can be expressed as

$$
I=2 \int_{0}^{\infty} \operatorname{drr} \rho(r),
$$

where $\rho(r)$ are phase-averaged coherent states (which correspond to a laser output well above threshold [1])

$$
\rho(r)=\frac{1}{2 \pi} \int_{2 \pi} d \phi|r \exp (i \phi)\rangle\langle r \exp (i \phi)|,
$$

and $|r \exp (i \phi)\rangle$ are coherent states $|\alpha\rangle$ with $\alpha=r \exp (i \phi)$. When illuminating the detector with phase-averaged coherent states $\rho(r)$ the measured statistics is essentially the phase average of the $Q$ function of $\Delta_{m}$,

$$
p_{m}(r)=\frac{1}{2} \int_{2 \pi} d \phi Q_{m}[r \exp (i \phi)] .
$$

After Eq. (A2) the desired $\operatorname{tr} \Delta_{m}$ can be determined by repeating the measurement for different input states $\rho(r)$ by suitably varying the coherent amplitude $r$

$$
\operatorname{tr} \Delta_{m}=\operatorname{tr}\left(\Delta_{m} I\right)=2 \int_{0}^{\infty} r d r p_{m}(r) .
$$

Although this recall a tomographic reconstruction of $\Delta_{m}$ [8], this is not the case since the illuminating state already carries the angular integration.

The same goal can be achieved following an slightly different strategy. This is by illuminating the detection system with thermal-chaotic states [Eq. (2.15)]. The corresponding $Q$ function [Eq. (2.17)] is a Gaussian centered at the origin of the complex plane and its width increases when the average mean number of photons $n_{\mathrm{tc}}$ increases. Thus for $n_{\mathrm{tc}}$ large enough the $Q$ function of the thermal-chaotic state will be approximately constant $Q(\alpha) \simeq Q_{0}$ on the area where $P_{m}(\alpha)$ $\neq 0$ so that the statistics will be proportional to $\operatorname{tr} \Delta_{m}$ :

$$
p_{m}=\pi \int d^{2} \alpha P_{m}(\alpha) Q(\alpha) \simeq \pi Q_{0} \int d^{2} \alpha P_{m}(\alpha)=\pi Q_{0} \operatorname{tr} \Delta_{m}
$$

and

$$
\operatorname{tr} \Delta_{m}=\lim _{n_{t c} \rightarrow \infty} \frac{p_{m}}{\pi Q_{0}}
$$


To illustrate this idea let us consider a one-photon detector whose output is contaminated by the vacuum and twophoton contributions

$$
\Delta_{1}=q|0\rangle\langle 0|+p| 1\rangle\langle 1|+q| 2\rangle\langle 2|,
$$

with $\operatorname{tr} \Delta_{1}=p+2 q$. When illuminated with the thermalchaotic state we get

$$
p_{1}=\frac{1}{n_{\mathrm{tc}}+1}\left[q+p \frac{n_{\mathrm{tc}}}{n_{\mathrm{tc}}+1}+q \frac{n_{\mathrm{tc}}^{2}}{\left(n_{\mathrm{tc}}+1\right)^{2}}\right] .
$$

For example, for $p=1$ and $q=0.1$ and taking $\pi Q_{0}=1$ / $\left(n_{\mathrm{tc}}+1\right)$, we get for $n_{\mathrm{tc}}=100$

$$
\operatorname{tr} \Delta_{1}=1.2, \quad \frac{p_{1}}{\pi Q_{0}}=1.19,
$$

i.e., only $1 \%$ error.
[1] L. Mandel and E. Wolf, Optical Coherence and Quantum Optics (Cambridge University Press, Cambridge, England, 1995); M. O. Scully and M. S. Zubairy, Quantum Optics (Cambridge University Press, Cambridge, England, 1997); V. V. Dodonov, J. Opt. B: Quantum Semiclassical Opt. 4, R1 (2002); U. M. Titulaer and R. J. Glauber, Phys. Rev. 140, B676 (1965); L. Mandel, Phys. Scr. T12, 34 (1986); D.-G. Welsch, W. Vogel, and T. Opatrný, in Progress in Optics, edited by E. Wolf (Elsevier, Amsterdam, 1999), Vol. 39; T. Richter and W. Vogel, Phys. Rev. Lett. 89, 283601 (2002); E. V. Shchukin and W. Vogel, Phys. Rev. A 72, 043808 (2005); R. Alicki and N. Van Ryn, J. Phys. A 41, 062001 (2008).

[2] M. Hillery, Phys. Rev. A 35, 725 (1987).

[3] H. J. Kimble, M. Dagenais, and L. Mandel, Phys. Rev. Lett. 39, 691 (1977); R. Short and L. Mandel, ibid. 51, 384 (1983); R. E. Slusher, L. W. Hollberg, B. Yurke, J. C. Mertz, and J. F. Valley, ibid. 55, 2409 (1985).

[4] D. N. Klyshko, Phys. Lett. A 213, 7 (1996); C. T. Lee, Phys. Rev. A 55, 4449 (1997).

[5] G. S. Agarwal and K. Tara, Phys. Rev. A 46, 485 (1992); A. Zavatta, V. Parigi, and M. Bellini, ibid. 75, 052106 (2007); T. Kiesel, W. Vogel, V. Parigi, A. Zavatta, and M. Bellini, ibid. 78, 021804(R) (2008).

[6] W. Vogel, Phys. Rev. Lett. 84, 1849 (2000); T. Richter and W. Vogel, ibid. 89, 283601 (2002); E. Shchukin, T. Richter, and W. Vogel, Phys. Rev. A 71, 011802(R) (2005); A. I. Lvovsky and J. H. Shapiro, ibid. 65, 033830 (2002).

[7] R. W. Spekkens, Phys. Rev. Lett. 101, 020401 (2008).

[8] A. Luis and L. L. Sánchez-Soto, Phys. Rev. Lett. 83, 3573 (1999); J. Fiurášek, Phys. Rev. A 64, 024102 (2001); H. B. Coldenstrodt-Ronge, J. S. Lundeen, K. L. Pregnell, A. Feito, B. J. Smith, W. Mauerer, Ch. Silberhorn, J. Eisert, M. B. Plenio, and I. A. Walmsley, J. Mod. Opt. 56, 432 (2009) ; J. S. Lundeen, A. Feito, H. Coldenstrodt-Ronge, K. L. Pregnell, Ch. Silberhorn, T. C. Ralph, J. Eisert, M. B. Plenio, and I. A. Walmsley, Nat. Phys. 5, 27 (2009).

[9] D. R. Cox and H. D. Miller, Theory of Stochastic Processes (Chapman \& Hall/CRC, London, 1977).
[10] L. Liu, J. Math. Anal. Appl. 328, 1484 (2007).

[11] S. Mancini, V. I. Man'ko, and P. Tombesi, Phys. Lett. A 213, 1 (1996).

[12] V. Bužek and P. L. Knight, in Progress in Optics, edited by E. Wolf (Elsevier, Amsterdam, 1995), Vol. 34, p. 1; C. C. Gerry and P. L. Knight, Am. J. Phys. 65, 964 (1997); M. Brune, S. Haroche, J. M. Raimond, L. Davidovich, and N. Zagury, Phys. Rev. A 45, 5193 (1992); L. Davidovich, A. Maali, M. Brune, J. M. Raimond, and S. Haroche, Phys. Rev. Lett. 71, 2360 (1993); M. Brune, E. Hagley, J. Dreyer, X. Maître, A. Maali, C. Wunderlich, J. M. Raimond, and S. Haroche, ibid. 77, 4887 (1996); L. Davidovich, M. Brune, J. M. Raimond, and S. Haroche, Phys. Rev. A 53, 1295 (1996); S. Haroche, Phys. Today 51 (7), 36 (1998); J. M. Raimond, M. Brune, and S. Haroche, Phys. Rev. Lett. 79, 1964 (1997); C. Monroe, D. M. Meekhof, B. E. King, and D. J. Wineland, Science 272, 1131 (1996); M. W. Noel and C. R. Stroud, Jr., Phys. Rev. Lett. 77, 1913 (1996); J. R. Friedman, V. Patel, W. Chen, S. K. Tolpygo, and J. E. Lukens, Nature (London) 406, 43 (2000).

[13] V. V. Dodonov, I. A. Malkin, and V. I. Man'ko, Physica 72, 597 (1974).

[14] H. P. Yuen and J. H. Shapiro, IEEE Trans. Inf. Theory IT-26, 78 (1980); B. Yurke, Phys. Rev. A 32, 311 (1985); U. Leonhardt and H. Paul, ibid. 48, 4598 (1993); T. Kim, Y. Ha, J. Shin, H. Kim, G. Park, K. Kim, T. G. Noh, and Ch. K. Hong, ibid. 60, 708 (1999).

[15] A. A. Semenov, A. V. Turchin, and H. V. Gomonay, Phys. Rev. A 78, 055803 (2008).

[16] For further connection between entanglement and classicality see for instance J. Sperling and W. Vogel, e-print arXiv:0811.4527.

[17] F. T. Arecchi, E. Courtens, R. Gilmore, and H. Thomas, Phys. Rev. A 6, 2211 (1972)

[18] O. Giraud, P. Braun, and D. Braun, Phys. Rev. A 78, 042112 (2008).

[19] A. Luis, Phys. Rev. A 73, 063806 (2006).

[20] A. Luis, Phys. Rev. A 66, 013806 (2002).

[21] A. Rivas and A. Luis, Phys. Rev. A 77, 022105 (2008). 